\title{
Patient Safety and Risk Management in Mental Health
}

\author{
Alessandro Cuomo, Despoina Koukouna, \\ Lorenzo Macchiarini, and Andrea Fagiolini
}

\section{Learning Objectives}

This chapter will review the most common adverse events that happen in a psychiatric unit and the safety measures that are needed to decrease the risk of errors and adverse events. The adverse events and errors that may happen in a psychiatric unit are unique and will be examined in detail. This section will also highlight the role of staff members and patients in preventing or causing the error.

A section of this chapter will describe the unique structural requirements that a psychiatric unit needs for optimal patient safety. The role of the doctors, nurses and staff to ensure safety will be discussed in detail.

\footnotetext{
A. Cuomo

Department of Molecular Medicine, University of Siena, Siena, Italy

D. Koukouna

Department of Molecular Medicine, University of Siena, Siena, Italy

L. Macchiarini

Private Practice, Bologna, Italy
A. Fagiolini $(\bowtie)$
Department of Molecular Medicine, University of Siena, Siena, Italy e-mail: andrea.fagiolini@unisi.it

\subsection{Introduction}

'Patient safety' refers to the prevention of harm, errors and adverse events to patients receiving healthcare. Despite the growing interest in patient safety, its application to mental health context is still largely unexplored [1]. Indeed, mental health hospitals or units face unique patient safety issues, such as those related to self-harm, suicide and use of restraints/seclusion [1,2].

The Institute of Medicine (US) categorizes patient safety events as either adverse events or medical errors [3]. An adverse event consists of an incident that causes harm to the patient and negatively affects the patient's health and quality of life, causing illness, injury, disability, suffering or death, and negatively affecting his or her social, physical or psychological structure [4]. A medical error is basically consisting of any mistake which is made during the diagnosis and treatment process [5].

The terms medical errors and adverse events are often used interchangeably. Medical errors can happen regardless of whether they lead to adverse events or cause no harm to the patient. Adverse events can be a consequence of a medical error but may well be also a result of appropriate care. For example, an event in which a patient, who has been appropriately assessed for not being at risk of a fall, accidentally trips and falls resulting in a fractured hip would be considered an adverse event. On the contrary, when a 
person who has been assessed to be at risk of a fall does not receive the prerequisite fall prevention implements, and then the person falls, the event is to be considered as a medical error even if the individual does not experience any injury [3].

\subsection{Epidemiology of Adverse Events in Patients Receiving Mental Healthcare}

Adverse events resulting from psychiatric conditions are influenced by various clinical, social and patient factors [3]. As for other disciplines, adverse events during mental healthcare may be described as unintended injuries caused by mental health management, resulting in an increased hospital stay or diminished function/disability at the time of discharge. Severe adverse events include those that result in the death of patients or permanent disability [6]. A system for categorizing events is needed to understand the multitude of factors leading to it [4]. Marcus et al. classified adverse events into nondrug-related events and medication-related events [3].

Nondrug adverse events include falls, assault, sexual contact, self-harm and other injuries [3].

\subsubsection{Nondrug-Related Adverse Events}

\subsubsection{Falls}

Falls in hospitals are common and can result in conditions ranging from prolonged hospitalization to death [7]. Falls in psychiatric units are more common than observed in many other medico-surgical units [8], and falls in geropsychiatric patients result in more severe outcomes [9].

According to the National Database of Nursing Quality Indicators, the rate of patient falls in psychiatric units is in the range of 13-25 per 1000 patient days, compared with 4 per 1000 patient days in medico-surgical areas [8]. The higher rate of falls in psychiatric inpatients is likely influenced by side effects of psychotropic medications, such as sedation, orthostatic hypertension [10] and medication-induced parkinsonism [9]. Lavsa et al. reported that the significant predictor of falls in the psychiatric population includes Alzheimer's disease, dementia, use of drugs like alpha-blockers, non-benzodiazepines sleep aids, benzodiazepines, $\mathrm{H}_{2}$ blockers, lithium, atypical and conventional antipsychotics, anticonvulsants and mood stabilizers, laxatives, and stool softeners [7]. Chan et al. found that adjustment or change in psychotropic drugs is also one of the major factors for falls in psychiatric units [9]. Other risk factors include orthostasis, gait instability, fluctuations in blood pressure and physical limitations [10]. All patients undergoing electroconvulsive therapy are assumed to be at a higher fall risk thereafter [11].

\subsubsection{Assault}

Assault refers to forcible physical contact and may include slapping, kicking, biting, punching and pulling hair. The assault need not result in an injury to be considered an adverse event. The exception is when a patient assaults a staff member, which is considered as an adverse event only if the staff member sustains an injury. No assaultive violence like the destruction of hospital property is usually not regarded as a patient safety event [3].

Studies have found that assaults, aggression or violence from a patient to staff members is more common than the same events directed towards another patient. Staggs found that higher levels of staffing by non-registered nurses resulted in higher rates of injurious assaults, regardless of who the victim (hospital personnel/other patients) was. Higher levels of staffing by registered nurses were associated with lower rates of injurious assaults against patients and higher rates of injurious assaults against hospital personnel [12]. A research conducted in a Norwegian psychiatric hospital showed that $100 \%$ of nurses had experienced a violent assault during their career [13].

Violence may cause an emotional effect like fear, anger, depression and sleeplessness on other inpatients. It can also result in increased absenteeism of staff members and high staff turnover [14]. Several negative variables that increase the 
risk for violence in mental hospitals have been identified in the interaction between inpatients and hospital staff [15] and multiple studies have identified variables such as extended hospital stay, previous episodes of aggression, and substance abuse, among the most significant predictors of violence in mental health units $[4,15]$.

\subsubsection{Sexual Contact}

Sexual contact is always considered as a patient safety adverse event when it is not voluntary. However, even voluntary sexual intercourses may be adverse events, given that patients admitted to an inpatient unit are often cognitively impaired and unable to give full consent. Also, risks may arise from the possibility of getting/ transmitting a sexually transmitted disease and/or of starting an unwanted pregnancy [3]. Sexual contact includes touching directly or indirectly, through the clothing, of the anus, breasts, genitalia, groin, buttocks and inner thigh. Sexual contact does not include non-physical contact such as sexual talk or non-sexual physical contact such as a pat on the back, hugging or kissing on the cheek greetings between a patient and a visitor, and where a staff member was an unwanted recipient of sexual contact from a patient [16]. Lawn and colleagues summarized a series of studies reporting that a high percentage of females experienced molestation or unwanted sexual comments during their stay in a psychiatric inpatient unit and that up to $56 \%$ of women reported having been troubled by men, with $8 \%$ stating that they had participated in sexual acts against their will. The authors note that consenting sexual activity on inpatient wards is a controversial issue but point to the fact that it is relatively common that inpatients have sexual intercourse. As an example, they report that in a study at Imperial College, $30 \%$ of the patients had engaged in some form of sexual intercourse, a percentage that was similar to the one (38\%) that was found in a survey of chronic patients in a facility of British Columbia, Canada [17].

\subsubsection{Self-Harm}

Psychiatric illnesses such as anxiety, depression and alcohol use disorders are well-known fac- tors for self-harm [18]. Self-harm has been described in many terms, including self-injury, deliberate self-harm, self-mutilation, attempted suicide or parasuicide [19]. People who selfinjure may be at an increased risk of suicide [18]. Exceptions include suicidal ideations that are not followed by actions to self-harm and minor injuries without any bruises, swelling or need for treatment [16].

A study conducted among acute inpatients found that the most common method of self-harm is ripping of the skin. The study also reported that men would more likely use outwardly aggressive methods to self-harm. Another study found out that among the inpatients who engage in selfharm episodes, women with no suicidal risk comprised the largest group. The chances of the patient trying to re-engage in self-harm are highest in the first 2 years after the first episode of self-harm, but the risk may persist over the next few years [19].

Patients who self-harm often describe feelings of upset, anger, loneliness, periods of inner tension, or feeling unreal, numbness or emptiness inside. James et al. cited psychological distress as the most common reason for self-harm. Studies found that environmental restriction increased the risk of self-harm. Other reasons for self-harm include refusal of a request by staff, feeling of being controlled by the staff, conflict with other patients and disappointment with the doctor [20].

\subsubsection{Other Nondrug Adverse Events}

These adverse events are usually caused by medical examination or treatment other than medications, such as electroconvulsive therapy [3]. The events may include difficulty in breathing or walking, seeing, hearing, or standing [16] and often result in stopping the treatment and causing functional impairments.

\subsubsection{Drug Adverse Events}

Adverse drug reaction (ADR) refers to the response of a drug that results in unintended and harmful consequences when the medication is 
given at doses that are typically used in humans for diagnosis, prevention or modification of physiological functions. These reactions result in increased mortality, morbidity, cost of treatment and non-adherence to treatment [21]. ADRs pose a significant problem in the treatment of patients with mental illness because these patients often lack adequate insight into their condition and treatment, and the ADR further complicates the situation [23]. Weight gain, constipation and tremors are among the most common ADRs that have been reported [21].

ADRs in psychiatry units are common and somewhat preventable. Rothschild et al. reported that $13 \%$ of all ADRs were preventable and atypical antipsychotics accounted for $37 \%$ of reported ADRs [22]. A study on the referrals of psychiatric inpatients to general hospitals found out that $76 \%$ of transfers were because of neurological reactions and $32 \%$ of transfers were because of the use of more than one psychotropic drug [23].

Studies conducted in New England and Kolkata, India, found that atypical antipsychotics were responsible for the majority of the ADRs reported in psychiatry units [21]. Thomas et al. conducted a study that involved the analysis and evaluation of ADRs reported in a psychiatric hospital for 3 years. The study found that the most frequent drugs associated with ADRs were antiepileptics, cardiovascular agents and secondgeneration antipsychotics. The study also found a $20.4 \%$ ADR preventability rate in the mental care units, which is lower than the preventability rates found in general inpatients and long-term facilities [24].

\subsection{Medical Errors in Psychiatric Care}

As in the general medical setting, medical errors in psychiatric care lead to significant injuries, up to death, as well as to an increase in the healthcare system costs.

Medical errors are classified into diagnostic errors, preventive errors, treatment errors and 'other errors'. Diagnostic failure includes failure to diagnose, including failure to order a diagnos- tic test. Preventive errors include failure in preventing disease or in monitoring the disease processes. Treatment errors include failure in providing medical interventions. Other errors include system error caused by a failure in the operating system or defective medical equipment.

The chain of events that leads to medical error is complex, but factors that lead to errors can be broadly classified as patient factors, provider factors and system factors.

\section{(a) Patient Factors}

Acute psychiatric symptoms such as impulsive, homicidal, suicidal and poorjudgement behaviour could result in either mistakes or slips. Mistakes include inaccurate treatment plans, whereas slips result from the deviation from the action plan. Psychiatric patients may not be able to accurately report their symptoms to the physician, which may delay treatment and complicate the differential diagnosis of diseases. Violent patients may induce distress and interfere with the decision-making process. For instance, even providers who are well knowledgeable of the dosing guidelines may end up administering excessive doses to these patients [25].

\section{(b) Provider Factors}

Mental healthcare providers have a considerable effect on patient safety [4]. Workplace stress and mental workload of the staff may impact on patients' safety and treatment. Communication deficiency is a commonly observed contributor to errors in psychiatry units [26]. Factors that may affect communication include fatigue, high staff turnover, lack of experience and interpersonal conflict [4]. Decreased length of stay by medical personnel in psychiatry units could raise the chances of missing out on crucial clinical information, such as medical comorbidities, medication allergies or medication dosing errors. The fear of aggression may result in an increased use of seclusion or restraint, as well as in insufficient therapeutic engagement [27]. 


\section{(c) System Factors}

Non-clinical systems such as training programmes, human resources, manualized admission and discharge processes are beyond the individual control of care providers but are relevant to patient safety [4]. Various factors pertaining to the general organization of mental healthcare delivery may have a significant effect on patient safety or lack thereof [4]. Institutional structures, operations and processes may be strained and vulnerable to errors [25].

Individuals with mental disorders are susceptible to the same type of errors that are seen in the general medical hospitals, such as diagnostic error, preventive error, treatment error and other errors.

\section{(a) Diagnostic Errors}

No biomarkers are available for the diagnosis of specific psychiatric conditions; therefore, the practitioner has to rely on subjective clinical assessment, to establish the diagnosis of the illness [28]. Establishing a diagnosis when adequate information about the patient's history is not available, especially of patients admitted on an emergency basis, is challenging and poses several risks. For instance, a missed diagnosis of bipolar disorder may lead to prescribing treatment with an antidepressant in mono therapy and result in agitation, impulse dyscontrol or manic switch.

\section{(b) Preventive Errors}

This type of error includes inadequate monitoring of patients and failure to provide prophylactic treatment. Inpatient psychiatric settings with inadequate protection systems such as locked wards and with a lack of 'nosharps' policies result in patient self-harm. Failure to monitor patients with suicidal risk may place patients in danger.

\section{(c) Treatment Errors}

Treatment errors include errors in administering treatment and medications, improper care and avoidable delays in initiating treatment. Another error in treatment is over sedation by handlers when the patient becomes aggressive or violent. Improper use of tranquillizers may over sedate patients which may result in severe patient falls.

\section{(d) Other Errors}

Other frequent errors include poor communication between patients, staff members and physicians. Effective communication between patients and service providers in psychiatric units is essential to understand patient history, conduct assessments and provide proper care [25].

\subsubsection{Common Errors and Dangerous Outcomes}

A summary of the most common errors associated with treatment in a mental health setting, as well as a review of their most dangerous outcomes, is provided below, with special reference to the errors that are associated with medication treatment, restraint, seclusion, and suicide.

\subsubsection{Medication Errors}

Medication errors are associated with an increase in morbidity and healthcare costs [29]. Medication administration error (MAE) may be the most commonly cited medication error in psychiatric hospitals. MAEs refers to a deviation from the instructions given by the psychiatrist, manufacturer and relevant institutional policies [26]. MAEs may occur due to failure in any of the five rights (right patient, dose, route, time and medication). These errors may arise both from mistakes committed by the provider and from system failures such as understaffing or inadequate prescribing or administration procedures [30].

\subsubsection{Restraint and Seclusion}

Restraint and seclusion, ordered to prevent aggressive patients from harming themselves, staff members and other patients, are controversial practices $[4,25]$. They are perceived by some as an infringement of human rights and by others as unavoidable last resource to maintain patient safety.

Restraint is categorized into environmental constraint, physical/mechanical constraint and 
chemical constraint. Environmental restraint (seclusion) refers to limiting the mobility of patients to a specifically designed and securely locked room. Physical/mechanical restraint refers to the use of any technique to manually restrict the free body movement of a person or any part of the body. Mechanical restraint refers to the use of appliances and devices like body vests, multiple-point ligatures, blankets and bedside rails to immobilize the patient. Chemical restraint refers to the use of drugs to rapidly tranquillize or sedate the patient. The drugs that are commonly used for this practice include benzodiazepines and antipsychotics [4].

Errors relating to restraint and seclusion primarily result from the misuse of restraints or holding techniques. Errors also result from failure in monitoring the patients from using sharp objects or cigarette lighter to relieve themselves from the restraint. Also, patients with comorbid illnesses like asthma, cocaine intoxication, cardiomyopathy, pulmonary hypertension or coronary artery disease are at an increased risk of sudden death by restraint devices. Failure in documenting and assessing these risk factors may affect patient safety [25].

\subsubsection{Suicide}

Suicide is described as deliberate physical selfharm leading to death [4]. Suicide attempts tend to peak during early admission to psychiatric institutes and soon after discharge [31]. As compared to the genera; population, the risk of dying by suicide while being admitted to a psychiatric unit is much higher. An English study reported that among the patients who died by suicide, 39\% were on agreed leave, $29 \%$ were absconding and $32 \%$ died in the ward [32]. Researchers have demonstrated that despite using sophisticated methods, it is impossible to predict suicide even in high-risk patients completely [11].

The most common predictors of inpatient suicide include a history of deliberate self-harm, depressive symptoms upon admission, and a diagnosis of schizophrenia [32]. The suicide incidences in patients who are suffering from schizophrenia are 10-13\% [33]. The method of suicide largely depends on the means that are available in the wards and includes hanging, strangulation, asphyxiation and cutting; among absconders the methods include drowning, jumping from a height, jumping in front of a vehicle and overdosing [31]. A large-scale study reported no difference in the risk of suicide in patients admitted to wards with and without doors [32]. However, the variables involved in suicide risk are multiple and there are situations in which an inpatient unit with closed doors is safer than a unit with open doors. Also, a closed unit avoids the need for nurses to spend time watching the door as opposed to talking and interacting with patients. However, there are situations in which a closed unit increases anxiety and agitation, over the feeling of being trapped, which might increase suicide risk. What is clear is that a poor assessment of suicide risk level and inadequate monitoring of patients at risk of suicide invariably leads to decreased safety [25].

\subsubsection{Nondrug Medical Error}

These include omitted, incorrect or delayed tests or procedures. They also include issues such as excessively low level of monitoring or observation, communication errors and failure to eliminate environmental dangers [16]. The three main categories of nondrug medical errors are errors proximal to the elopement, errors proximal to contraband and other errors [3].

\subsubsection{Errors Contributing to Elopement}

Elopement is the unauthorized absence of a patient from the psychiatric unit. Common reasons for elopement include the feeling of being trapped/confined, desire to use drugs or alcohol, feeling to be cut off from family and friends, boredom, being afraid of the other patients, objection over taking medications and stigma about being in a psychiatric unit. Involuntarily admitted patients have a higher chance of eloping from the psychiatric unit [34]. Elopement by itself is not an error, but the use of unguarded doors or windows may be considered as an error which allows the patient to flee from the unit [3]. 


\subsubsection{Errors Contributing to Contraband}

Contrabands are potentially hazardous items prohibited in the psychiatry unit. These include rope-like items such as shoelaces, headphone wires and belts; sharp objects such as scissors and razors; matches and lighters, illegal drugs and alcohol; plastic bags and balloons [16]. Detection of contraband is not an error, but a partial search of the body and belongings of the patients and visitors in the psychiatric unit is an error [3].

\subsubsection{Other Errors}

These include any other error that occurs during the hospitalization. Examples include performing a wrong or undue test or procedure, not performing test or procedures that were ordered, or issues such as administering food to a patient known to be allergic to that specific food [3].

\subsection{Safety Practices and Implementation Strategies}

Inpatient psychiatric care is often necessary to administer medications and procedures that cannot be administered on an outpatient basis. However, admission to an inpatient unit may also be due to the need for other reasons, such as the need to protect a patient from dangerous behaviours [16].

The most common strategies to reduce medical errors and adverse events and to provide a safe and therapeutic environment for inpatient psychiatric care are reported below.

\subsubsection{Role of the Hospital Environment in Patient Safety}

The unique structure of psychiatric ward plays a vital role in patient safety. Replacing the hazardous things in the psychiatric ward with nonhazardous materials may help in preventing the harm caused by the patient to self or others [35]. Avoiding anchors for ligatures and materials used for ligatures during construction or renovation of a psychiatric unit may help to prevent many adverse events [11]. Ligature anchor points are protrusions capable of supporting body weight for patients who hang themselves [31].

The most common ligature points are hooks or handles, doors, curtain rods, closet clothing rods, towel rods and sprinkler heads [31, 36]. The facility should be regularly inspected, given that the bathroom, closet, bedroom, and a hidden area of a unit are places where patients can selfharm [11]. Doors should be removed from places where not required. It has been reported that the number of suicides following absconding from a locked door was the same as those that occurred in open wards [31]. Door hinges should be in the continuous piano style from the top to the bottom of the door, in an unbroken manner, to avoid that patients to tie the cord to the hinge [36]. Wardrobe cabinets should not have doors, and hangers and rods should be replaced with shelves.

Exposed utility pipes should be covered. Fire safety sprinklers and showerheads should have a flush-mounted design. Shower curtain rods should be removed or designed such that they break away even when a least-heavy patient tries to commit suicide. Shower controls, sink faucets and handles should be such that they will not support a cord. A rounded design can be incorporated. Plastic or unbreakable windows and mirrors should be used throughout the unit. Dining utensils should not be able to cause selfinjury or to others. The dishes should also be unbreakable.

Patients can also use structures close to the floor for asphyxiation. Fixtures close to the floor should have a design such that they do not support a strangulation device. Items that can be used by patients for strangulation or hanging should be avoided inside the unit. These items include handkerchiefs, ties, belts, drawstring belts and shoelaces. Because patients can also use straps associated with bras for strangulation, it is necessary to assess women patients who are at a high risk of suicide and consideration should be made whether such women should be allowed to wear a bra [36]. 
The use of latest technology for safety, security and medicine administration helps in the safety of patients. The use of security system helps the patients feel well protected and protects them from unwanted visitors, robbery and access to alcohol and drugs [35].

\subsubsection{Role of Organizational Management in Patient Safety}

Organizational management plays a major role in patient safety [37]. The time spent by hospital managers and board of directors to visit and inspect the inpatients units positively influence safety performance [38]. Leadership comprises three areas: availability, experience and understanding patient safety plan. The role of leadership is to enhance learning, teamwork, feedback and improvement in individual worker's safety behaviour. They provide support for the staff and help in creating a good working environment. Also, if any adverse event occurs, they act as a support system for the staff [37].

\subsubsection{Role of Staff in Patient Safety}

Nurses play an important role in patient safety, especially in inpatient psychiatric wards [37]. According to a study conducted by Ajalli et al., the role of nurses is defined in two terms, 'close observing' and 'vigilant care' [39]. The head nurse plays an important role in encouraging patient safety culture among other nursing staff. The head nurse should do the following: communicate effectively, contribute to leadership, maintain a positive culture and provide patient-centred care. The presence of nurses with experience in dealing with psychiatric patients is strongly connected to the high quality of patient care and to better outcomes [35].

The staff members should possess skills to help with the disorders that are being treated in the unit, and skills to help patients with self-care. They should be able to provide reassurance, and basic information and knowledge about the ill- ness, the treatment and its administration procedures. Also, they should be able to identify and address trauma-related needs [37].

Staff load is also a concern in patient safety. The number of patients is assigned to each nurse and staff member depending up on many factors which include the experience and qualification of the staff and patient's condition. Nurses who lack experience in dealing with patients with mental illnesses negatively impact the quality of care provided and this should be accounted for when the number of persons allocated to a shift is decided. Also, patients that are better stabilized should preferably be assigned to the nurses with less experience [35].

Staff safety, health and well-being are important in dealing with psychiatric patients [35, 37]. Hospital administration should take care that staff do not suffer from stress, fatigue or distraction from work. Staff members should have a healthy state of mind to be able to help patients experiencing an acute mental disease.

Staff members need to have good communication skills (written and verbal) to understand the patient's condition and minimize errors. Failure in communication is a known source for adverse events. Appropriate communication is also important during the transfer of a patient as an error in transferring the medical and psychiatric history may lead to serious adverse events. Communication also helps patients to seek help and safety when they feel a threat [37].

\subsubsection{Role of the Patient in Patient Safety Practices}

Involving patients in safety issues is key to reducing risks and preventing errors [37]. Establishing a safe nurse-patient environment is important for effective patient care, and helps to better understand the patient's needs $[35,37]$. Whenever possible, patient's preference should be considered so that the decision made aligns with the patient's values. Patient's health condition and personality also play an important role because it influences communication and interest to become involved in the care [37]. 
Research has found that inpatients are active in making their environment safer for themselves by avoiding excessive interactions with risky individuals or situations, by contributing to deescalating potential risky situations, and seeking surveillance or other safety interventions from staff. These findings underline the importance of fully involving patients in safety initiatives [4].

\subsection{Conclusion}

Several adverse events and medical errors are unique to inpatient psychiatric care. Several safety measures are able to decrease the risk of errors and adverse events. The most important strategies to improve safety in mental health facilities include an appropriate consideration of the structural requirements that a psychiatric unit needs to have in place to ensure the optimal patient safety. Also, administrators, doctors, nurses and staff play a proactive role to ensure safety. Whenever possible, patients themselves should be involved in procedures able to increase their safety.

\subsection{Case Studies}

\section{The case examples below are fictitious and for didactic purposes only}

\subsubsection{Case Example 1}

Mr. Mario, a 40-year-old man, was admitted to the emergency room, accompanied by his parents, for his 'strange and anomalous' behaviour in the last days. He lived with his parents and is their only child. He unemployed and had no sentimental ties.

Family members traced the onset of his symptoms when he was approximately 20 years old. At the time, Mario was a university student and began to manifest the bizarre symptoms and behaviours. He reduced the time spent in class and the relationships with his peers. He spent most of his time isolated in his room. He slept through the entire day and stayed awake at night. After a few months, the situation worsened, and he stopped communicating with everyone, including his parents, appeared confused, anguished and disturbed. He neglected basic needs such as personal hygiene and eating. One day he barricaded himself in his room, refusing to leave because he was convinced that the mafia had spies and cameras all over his life. His parents took him to a hospital, where an antipsychotic drug therapy was started, and a diagnosis of paranoid schizophrenia was made at discharge after a few days in the inpatient unit. Over the years, he was relatively stable, but his insight remained limited and his parents had to constantly convince him taking the prescribed treatment.

Six months ago, Mario's mother was diagnosed with breast cancer. She was initially hospitalized to undergo mastectomy and later received chemotherapy. This situation resulted in the reduced presence of parents at home and decreased her support in Mario's everyday life. Mario stopped his medication without informing neither the parents nor the reference psychiatrist. Persecutory delusions reappeared; he spent all his time locked in his room, had no relationship with anyone, remained naked in the dark and neglected his basic needs.

Mario was admitted to inpatient care and restarted being treated with an antipsychotic. In the following days, he improved and went gradually towards remission. His parents visited and spend time with him every day. After 5 days, however, the mother was hospitalized because of a severe infection and could no longer visit her son. Mario did not verbalize his concern for his mother's health but refused to talk and was apathetic. He was no longer a participant in the activities of the psychiatry unit and began to show indifference to everything and everyone, including his father who visited him regularly.

One night, when the health workers were busy to admit two new patients in the psychiatry department, Mario managed to steal the belt of a newly arrived patient and tried to hang himself in the shower of his room. Fortunately, the patient with whom he shared the room noticed what was 
happening and promptly warned the nurses who intervened and saved Mario's life.

\subsubsection{Discussion}

This case outlines that the level of stability and the required level of care may quickly change during the periods of inpatient admission. It is a reminder for the need to talk to the patients, check their suicidal ideation and constantly monitor their level of stability. Also, it reminds us about the need to not decrease the level of attention on the other patients when new patients are admitted to the inpatient unit. Finally, it reminds us of the key role of other patients in increasing their and other patients' level of safety.

\subsubsection{Case Example 2}

Ms. Alice was a patient admitted to the geriatric psychiatric unit because of a severe major depressive episode.

She is 78 years old. Her married daughter lived in another city. She suffered from arterial hypertension that was being treated pharmacologically with ACE inhibitor (ramipril $5 \mathrm{mg}$ /day). She was also taking an oral opioid analgesic (tramadol $200 \mathrm{mg} /$ day), for the past 2 months, to cope with severe pain and functional limitation of the right knee associated with medial femoraltibial gonarthrosis. After her husband passed away 3 months ago, Ms. Alice did not sleep well at night and did not eat much. She preferred staying in bed most of the day and no longer seemed interested in any of her usual daily activities. Two weeks earlier, a general practitioner prescribed her antidepressant therapy with sertraline, which was started at $50 \mathrm{mg} /$ day but did not lead to any improvement in her symptoms.

Upon admission, her sertraline was increased to $100 \mathrm{mg} / \mathrm{day}$.

Five days after, she started to present with tremors, increased sweating, confusion, hyperpyrexia $\left(39^{\circ} \mathrm{C}\right)$, tachycardia, arterial pressure of $170 / 80 \mathrm{mmHg}$ and respiratory rate of 30 acts/ min. She also presented with chills, diarrhoea, vomiting, mydriasis and marked neuromuscular hyperactivity (more prominent in the lower extremities) with tremor plus hyperreflexia, hypertonia and muscle stiffness, myoclonus, spontaneous clonus and plantar extensor responses.

Based on history taking, laboratory and physical examination, Alice was diagnosed with serotonin syndrome. Treatment with tramadol and sertraline were stopped, and the patient was hydrated with intravenous fluids. The mental state of the patient improved within $24 \mathrm{~h}$.

The hospital regime diagnostic-instrumental investigations excluded the presence of other organic pathologies of a neurological, infective or metabolic nature.

Upon discussion of the case, it turned out that the staff members had noticed-since her first day of admission - that she had sweating, hypertonia and tremors while drying her off after the daily shower. However, they did not report this finding to the attending nurses and physician.

\subsubsection{Discussion}

It is imperative to pay attention when a patient is taking a selective serotonin reuptake inhibitors (SSRIs), tramadol, in addition to a CYP2D6 inhibitor. These isoenzymes are involved in extensive metabolism of SSRIs in the liver. The CYP2D6 system, one of the key enzymes involved in adverse drug reactions, has a high degree of genetic polymorphism. Studies cite that $7 \%$ of white patients lack the capacity to metabolize drugs by CYP2D6 enzyme. Consequently, the serum levels of tramadol would be higher; and such patients would be at an increased risk for serotonin syndrome if a second serotonergic agent is added to the pharmacological protocol. The attending physician who admitted the patient likely made an error in continuing the medications that the patient was taking at home. The attending physician in the unit likely made an error in increasing the dose of sertraline, without considering the interaction with tramadol and without carefully examining the patient for symptoms of the serotonin syndrome. The staff members that noticed her tremor, sweating and hypertonia likely made an error in not communicating the above to the nurses and psychiatrists. A careful evaluation of the interactions of the medi- 
cations that are prescribed in combination, a thorough physical exam conducted before and after raising the dose of sertraline, and a more appropriate communication to the nurses and physician of the observations from staff members could have prevented the onset of a potentially fatal event.

\section{References}

1. D'Lima D, Crawford MJ, Darzi A, Archer S. Patient safety and quality of care in mental health: a world of its own? BJPsych Bull. 2017;41(5):241-3.

2. Brickell TA, McLean C. Emerging issues and challenges for improving patient safety in mental health: a qualitative analysis of expert perspectives. J Patient Saf. 2011;7(1):39-44.

3. Marcus SC, Hermann RC, Cullen SW. Defining patient safety events in inpatient psychiatry. J Patient Saf. 2018; https://doi.org/10.1097/ PTS.0000000000000520.

4. Brickell AT, et al. Patient safety in mental health. BC Mental Health and Addiction Services, p. 9, 16, 12.

5. Medication errors and patient safety in mental health. [Internet]. Available from: https://www.medscape. org/viewarticle/563039.

6. Jayaram G. Measuring adverse events in psychiatry. Psychiatry (Edgmont). 2008;5(11):17-9.

7. Lavsa SM, Fabian TJ, Saul MI, Corman SL, Coley KC. Influence of medications and diagnoses on fall risk in psychiatric inpatients. Am J Health Syst Pharm. 2010;67(15):1274-80.

8. Abraham S. Managing patient falls in psychiatric inpatient units: Part 2. Health Care Manag (Frederick). 2016;35(2):121-33.

9. Chan CH, Gau SS, Chan HY, Tsai YJ, Chiu CC, Wang SM, Huang ML. Risk factors for falling in psychiatric inpatients: a prospective, matched case-control study. J Psychiatr Res. 2013;47(8):1088-94.

10. Khurshid T, Lantz MS. Falls and inpatient geriatric psychiatry: a simple solution to a chronic and difficult problem. Am J Geriatr Psychiatry. 2016;24(3):S90-1.

11. Burke WJ, Rubin EH, Zorumski CF, Wetzel RD. The Safety of ECT in Geriatric Psychiatry. J Am Geriatr Soc. 1987;35(6):516-21.

12. Staggs VS. Injurious assault rates on inpatient psychiatric units: associations with staffing by registered nurses and other nursing personnel. Psychiatr Serv. 2015;66(11):1162-6.

13. Quirk A, Lelliott P, Seale C. Service users' strategies for managing risk in the volatile environment of an acute psychiatric ward. Soc Sci Med. 2004;59(12):2573-83.

14. Iozzino L, Ferrari C, Large M, Nielssen O, de Girolamo G. Prevalence and risk factors of violence by psychiatric acute inpatients: a systematic review and meta-analysis. PLoS One. 2015;10(6):e0128536.

15. Cornaggia CM, Beghi M, Pavone F, Barale F. Aggression in psychiatry wards: a systematic review. Psychiatry Res. 2011;189(1):10-20.

16. Marcus SC, Hermann RC, Frankel MR, Cullen SW. Safety of psychiatric inpatients at the veterans health administration. Psychiatr Serv. 2018;69(2):204-10.

17. Lawn T, McDonald E. Developing a policy to deal with sexual assault on psychiatric in-patient wards. Psychiatr Bull. 2009;33:108-11.

18. Singhal A, Ross J, Seminog O, Hawton K, Goldacre MJ. Risk of self-harm and suicide in people with specific psychiatric and physical disorders: comparisons between disorders using English national record linkage. J R Soc Med. 2014;107(5):194-204.

19. Tofthagen R, Talseth AG, Fagerstom L. Mental health nurses' experience of caring for patients suffering from self harm. Nurs Res Pract. 2014;2014:905741.

20. James K, Stewart D, Bowers L. Self-harm and attempted suicide within inpatient psychiatric services: a review of the literature. Int J Ment Health Nurs. 2012;21(4):301-9.

21. Chawla S, Kumar S. Adverse drug reactions and their impact on quality of life in patients on antipsychotic therapy at a tertiary care center in Delhi. Indian J Psychol Med. 2017;39(3):293-8.

22. Rothschild JM, Mann K, Keohane CA, Williams DH, Foskett C, Rosen SL, Flaherty L, Chu JA, Bates DW. Medication safety in a psychiatric hospital. Gen Hosp Psychiatry. 2007;29(2):156-62.

23. Popli AP, Hegarty JD, Siegel AJ, Kando JC, Tohen M. Transfer of psychiatric inpatients to a general hospital due to adverse drug reactions. Psychosomatics. 1997; 38(1):35-7.

24. Thomas M, Boggs AA, DiPaula B, Siddiqi S. Adverse drug reactions in hospitalized psychiatric patients. Ann Pharmacother. 2010;44(5):819-25.

25. Nath SB, Marcus SC. Medical errors in psychiatry. Harv Rev Psychiatry. 2006;14(4):204-11.

26. Keers RN, Plácido M, Bennett K, Clayton K, Brown P, Ashcroft DM. What causes medication administration errors in a mental health hospital? A qualitative study with nursing staff. PLoS One. 2018;13(10):e0206233.

27. Slemon A, Jenkins E, Bungay V. Safety in psychiatric inpatient care: the impact of risk management culture on mental health nursing practice. Nurs Inq. 2017;24(4):e12199.

28. Phillips J. Detecting diagnostic error in psychiatry. Diagnosis (Berl). 2014;1(1):75-8.

29. Maidment ID, Parmentier H. Medication error in mental health: implications for primary care. Ment Health Fam Med. 2009;6(4):203-7.

30. Medication administration errors. [Internet] [Sept 2019]. Available from: https://psnet.ahrq.gov/primer/ medication-administration-errors.

31. Sakinofsky I. Preventing suicide among inpatients. Can J Psychiatr. 2014;59(3):131-40. 
32. Madsen T, Erlangsen A, Nordentoft M. Risk estimates and risk factors related to psychiatric inpatient suicide - an overview. Int J Environ Res Public Health. 2017;14(3):253.

33. Hussein ZN, Solomon H, Yohannis Z, Ahmed AM. Prevalence and associate factors of suicide ideation and attempt among people with schizophrenia at Amanuel Mental Specialized Hospital, Addis Ababa, Ethiopia. J Psychiatry. 2015;18:1.

34. Brumbles D, Meister A. Psychiatric elopement: using evidence to examine causative factors and preventative measures. Arch Psychiatr Nurs. 2013;27(1):3-9.

35. Alshowkan A, Gamal A. Nurses' perceptions of patient safety in psychiatry wards. IOSR J Nurs Health Sci. 2019;8(1):3.
36. Lieberman DZ, Resnik HLP, Holder-Perkins V. Environmental risk factors in hospital suicide. Suicide Life Threat Behav. 2004;34(4):448-53.

37. Kanerva A, Lammintakanen J, Kivinen T. Patient safety in psychiatric inpatient care: a literature review. J Psychiatr Mental Health Nurs. 2012;20(6):541-8.

38. Parand A, Dopson S, Renz A, Vincent C. The role of hospital managers in quality and patient safety: a systematic review. BMJ Open. 2014;4:e005055.

39. Ajalli A, Fallahi-Khoshknab M, Hosseini MA, Mohammadi E, Nir MS. Explanation of patient safety provided by nurses in inpatient psychiatric wards in Iran: a qualitative study. Iran J Psychiatry Behav Sci. 2018;12(4):e67951.

Open Access This chapter is licensed under the terms of the Creative Commons Attribution 4.0 International License (http://creativecommons.org/licenses/by/4.0/), which permits use, sharing, adaptation, distribution and reproduction in any medium or format, as long as you give appropriate credit to the original author(s) and the source, provide a link to the Creative Commons license and indicate if changes were made.

The images or other third party material in this chapter are included in the chapter's Creative Commons license, unless indicated otherwise in a credit line to the material. If material is not included in the chapter's Creative Commons license and your intended use is not permitted by statutory regulation or exceeds the permitted use, you will need to obtain permission directly from the copyright holder. 